\title{
Right ventricular diastolic relaxation in conscious dog models of pressure overload, volume overload, and ischemia
}

\author{
Ares D. Pasipoularides, MD, PhD \\ Ming Shu, PhD \\ Ashish Shah, MD \\ Donald D. Glower, MD
}

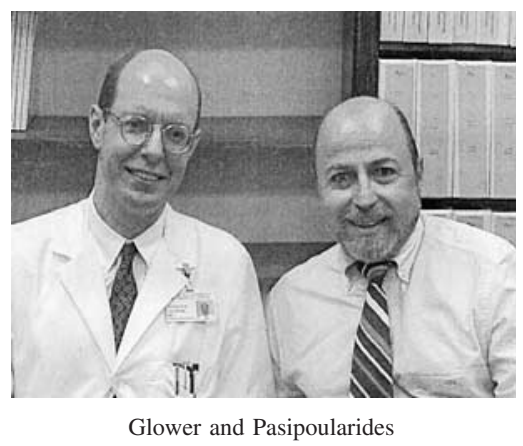

From the Department of Surgery, Division of Cardiothoracic Surgery, and the Duke Center for Emerging Cardiovascular Technologies, Duke University, Durham, NC.

This work was supported in part by National Heart, Lung, and Blood Institute grant R01 HL-50446 (Dr Pasipoularides) and the Duke/NSF ERC for Emerging Cardiovascular Technologies.

Received for publication Nov 6, 2001; revisions requested $\mathrm{Feb}$ 5, 2002; revisions received April 4, 2002; accepted for publication May 28, 2002.

Address for reprints: Donald D. Glower, MD, Division of Cardiac and Thoracic Surgery, PO Box 3851, Medical Center, Duke University, Durham, NC 27710 (E-mail: glowe001@mc.duke.edu).

J Thorac Cardiovasc Surg 2002;124:964-72

Copyright $(\odot) 2002$ by The American Association for Thoracic Surgery

0022-5223/2002 $\$ 35.00+0 \quad \mathbf{1 2 / 1 / 1 2 6 6 7 7}$

doi: $10.1067 / \mathrm{mtc} .2002 .126677$
Objective: Limitations in clinical understanding of right ventricular relaxation can be attributed to the paucity of information from basic studies in animal models of right ventricular disease. This study examined, in the conscious state, right ventricular relaxation dynamics under normal conditions $(n=15)$ and in subacute $(2-5$ weeks) canine models of right ventricular pressure overload $(n=6)$, volume overload $(n=7)$, and free wall ischemia $(n=7)$.

Methods: Right-heart micromanometric measurements were obtained by using multisensor catheters. A new algorithm was developed to obtain representative ensemble averages of hemodynamic waveform data sets. Right ventricular relaxation was analyzed by using an exponential model with 3 parameters: $\mathrm{P}_{0}, \tau$, and $\mathrm{P}_{\mathrm{b}}$. Significant changes versus control values were determined by means of analysis of variance and the Student unpaired $t$ test with Bonferroni's adjustment.

Results: In the state of pressure overload, right ventricular pressure decay exhibits an increased $\mathrm{P}_{0}(56.2 \pm 19.1 \mathrm{vs} 13.1 \pm 5.1 \mathrm{~mm} \mathrm{Hg}$ [mean $\left.\pm \mathrm{SD}]\right)$ and prolonged $\tau(57.1 \pm 2.8$ vs $27.8 \pm 3.9 \mathrm{~ms})$; there is also a decreased $\mathrm{P}_{\mathrm{b}}(-7.9 \pm 1.5$ vs $0.28 \pm$ $1.8 \mathrm{~mm} \mathrm{Hg}$ ). The only significant change in volume overload is an increased asymptote, $\mathrm{P}_{\mathrm{b}}(5.3 \pm 2.9 \mathrm{~mm} \mathrm{Hg})$. In right ventricular ischemia, prolongation of $\tau$ $(41.4 \pm 13.0 \mathrm{~ms})$ and decreased $\mathrm{P}_{\mathrm{b}}(-1.95 \pm 1.1 \mathrm{~mm} \mathrm{Hg})$ attain high significance.

Conclusions: Distinctive abnormalities in right ventricular relaxation dynamics accompany pressure overload, volume overload, and ischemia and may contribute to clinical right ventricular dysfunction.

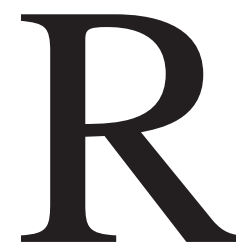

ight ventricular (RV) dysfunction is implicated in nearly $20 \%$ of all deaths associated with congestive heart failure, ${ }^{1-4}$ and the right ventricle plays a crucial role in the cardiopulmonary interactions of many disease states.1,2 Moreover, RV dysfunction is often the limiting factor for the success of coronary bypass surgery, heart transplantation, or heart-lung transplantation.., 6

Despite its physiologic and clinical importance, however, little attention has been paid to relaxation abnormalities, specifically those of the right ventricle. ${ }^{1-4,6}$ Limitations of our understanding can be attributed to the paucity of information from basic studies of RV relaxation in appropriate animal disease models. The aim of the present study was to study RV relaxation dynamics in subacute animal models of $\mathrm{RV}$ pressure overload, RV volume overload, and RV free wall ischemia.

\section{Methods}

All animals received humane care in compliance with the "Guide for the Care and Use of Laboratory Animals" prepared by the Institute of Laboratory Animal Resources, National Research Council, and published by the National Academy Press, revised 1996. 


\section{Acquisition of Control Data}

Lying on its right side, each dog (20-30 kg) was sedated with morphine $(0.7 \mathrm{mg} / \mathrm{kg})$; dosage was adjusted so as to sedate the animal while keeping it awake.

A $30-\mathrm{cm}, 8 \mathrm{~F}$ sheath introduced through a jugular cutdown allowed passage of a custom RV catheter (Millar Instruments, Inc, Houston, Tex). Mounted on the catheter were 2 micromanometers, $5 \mathrm{~cm}$ apart, with the distal one at the tip. The micromanometers were soaked in saline solution at $36^{\circ} \mathrm{C}$ to $38^{\circ} \mathrm{C}$ for a minimum of 3 hours; they were simultaneously balanced and calibrated immediately before each study.

Once proper functioning of micromanometers had been verified, the RV catheter was advanced during fluoroscopy until the proximal and distal micromanometers were located inside the right atrium and ventricle, respectively. When a steady-state physiologic condition was reached, pressure data were obtained and digitized at $400 \mathrm{~Hz}$.

Multiple data sets were recorded under control conditions and under each disease state. Each set was between 20 and 30 seconds to ensure sufficient numbers of beats for analysis.

\section{Induction of RV Pressure Overload}

Dogs (20-30 kg) were premedicated with cefazolin $(500 \mathrm{mg})$ and iron dextran $(100 \mathrm{mg})$ and anesthetized with intravenous pentobarbital $(20 \mathrm{mg} / \mathrm{kg})$ and succinylcholine $(1 \mathrm{mg} / \mathrm{kg})$. They were intubated and ventilated with a Bennett MA 1 respirator (PuritanBennett, Los Angeles, Calif). Thoracotomy was performed under sterile conditions through the left fifth intercostal space, exposing the heart and major vessels. RV pressure overload was induced through pulmonary artery (PA) occlusion by using a silicone rubber pneumatic occluder around the main PA. The trunk beneath the occluder was wrapped with polytetrafluoroethylene to prevent PA rupture. Pressure overload was induced by inflating the PA occluder until peak RV systolic pressure reached at least $60 \mathrm{~mm}$ $\mathrm{Hg}$ or approximately twice its control level. The occluder remained inflated throughout the study period. The occluders were filled with hypertonic glycerin solution. This kept balloon volumes very stable; few leaked, and a few even swelled by drawing in fluid. We recorded the inflation volume and checked the balloon volume weekly to maintain inflation. If any leak was detected, the degree of occlusion was readjusted by either echocardiography or catheterization. An additional check was on the volume of glycerin in the balloon, which could be briefly deflated and then reinflated with the original volume found to provide the desired gradient. At the time of the study, echocardiography and right-heart catheterization confirmed stable PA stenosis. Pressure overload data were collected between the third and fifth weeks after its induction.

\section{Induction of RV Volume Overload}

Tricuspid regurgitation (TR) was used for volume overload. The right external jugular vein was exposed after achievement of local anesthesia with $1 \%$ lidocaine, and an $8 \mathrm{~F}, 25-\mathrm{cm}$ introducer sheath was positioned into the right ventricle under fluoroscopy. A $6 \mathrm{~F}$ urologic biopsy forceps (Circon Instruments, Santa Barbara, Calif) was inserted into the right ventricle through the sheath, and multiple passes were taken to sever chordae until 3 to $4+$ regurgitation developed. Regurgitation severity was confirmed by means of contrast fluoroscopy and central venous pressure. TR was achieved such as to yield both complete filling of the atrium with contrast medium within several cycles and an increase of peak right atrial pressures (V wave) to greater than $15 \mathrm{~mm} \mathrm{Hg}$ with obliteration of the X-Y descent. This model produced consistent RV dysfunction, with dilatation at 1 week and ensuing reproducible failure. Hemodynamic data were collected between the second and third week after TR.

\section{Induction of RV Free Wall Ischemia}

A left lateral thoracotomy was performed after achievement of general anesthesia, as described earlier. An open chest greatly enhanced the surgeon's ability to produce controllable ischemia associated with only the RV free wall, without affecting the LV. A 50-mg dose of intravenous lidocaine was administered, and the right coronary artery was ligated. After stabilization, multiple RV branches off the left anterior descending and posterior descending coronary arteries were also ligated to limit collateral flow. Hemodynamic data were collected in the second week after the procedure. At the time of autopsy, the condition of ischemia was assessed by means of visual inspection of the infarcted myocardium. Ischemia was confirmed by means of histopathologic staining (hematoxylin and eosin), showing a $30 \%$ or greater infarcted cross-sectional myocardial region of the RV free wall.

\section{Analysis of Cardiodynamic Data}

Data processing involved determination of steady-state beats and ensemble averaging to obtain a waveform representative of any given measurement set. Because RV pressure in conscious animals is strongly influenced by respiration, ${ }^{7}$ data were averaged over several respiratory cycles. Furthermore, compared with each of its constituent waveforms, the ensemble average has a higher signal/ noise ratio proportional to the square root of the number of pulses used. Hemodynamic signal processing was performed with custom software developed in our laboratory. It included the following steps: conversion of any given data file into standard format; separation of individual beats; selection of steady-state beats; length adjustment of individual beats; ensemble average calculation; and modeling of RV relaxation dynamics.

Conversion of data into standard format. Each standard data file contained values for right atrial pressure and RV pressure and its change with respect to time $\left(R V \frac{d p}{d t}\right) \cdot R V \frac{d p}{d t}$ was computed digitally from the micromanometric pressure data.

Separation of beats. The beginning of a beat was defined at the onset of RV relaxation, occurring at the nadir of RV pressure change with respect to time. ${ }^{8,9}$ The software needed to detect a period of continuous pressure decline of at least $150 \mathrm{~ms}$ before locating maximum value of $\left(-\frac{d p}{d t}\right)$ to reduce the effect of confounding transients associated with outflow deceleration and aortic valve closure. ${ }^{10}$ The end point of one beat coincides with the start of the next. After an entire train had been processed, the beginning and length of each beat were recorded.

Selection of steady-state $\boldsymbol{R} \boldsymbol{V}$ pulses A subset of the individual beats from the previous step was selected for ensemble averaging. Whether the individual waveforms used are truly representative of the physiologic steady state is the factor determining the quality of the resulting average. It was therefore required that the SD of the 
TABLE 1. Statistics on length variation of hemodynamic waveforms

\begin{tabular}{lcccr}
\hline & Control & P0 & V0 & Ischemia \\
\hline Mean length (ms) & 550 & 480 & 530 & 435 \\
Maximum length (ms) & 700 & 595 & 640 & 14 \\
Mean SD (ms) & 12 & 17 & 22 & 16 \\
Maximum SD (ms) & 18 & 24 & 2.8 & 25 \\
Mean SD/length (\%) & 2.4 & 3.3 & 3.9 & 4.6 \\
Maximum SD/length (\%) & 3.1 & 4.2 & 4.7 \\
\hline
\end{tabular}

$S D$, Standard deviation; $P O$, pressure overload; VO, volume overload.

length of selected steady-state beats be within less than $5 \%$ of the average length. Table 1 shows that the beat ensembles used contain beats that are very similar in length. The SDs of these ensembles are between $2 \%$ and $5 \%$ of the mean length.

Standardization of beat length and ensemble averaging. Ensemble averaging requires each individual beat to have the same length. A new expansion-contraction (EC) algorithm adjusted all selected waveforms to the mean length of their ensemble while maintaining all the shape characteristics of the constituent waveforms. The EC algorithm normalizes all constituent beats to a standard length before averaging, as opposed to another algorithm that truncates or extrapolates (TE) to offset individual beat-length differences.

The EC algorithm is based on recreating each beat in an ensemble by using an adjustable sampling frequency. The number $\left(\mathrm{N}_{\mathrm{av}}\right)$ of data points in a pulse of the average length, $\mathrm{t}_{\mathrm{av}}$, is given by the following formula:

$$
\mathrm{N}_{\mathrm{av}}=\mathrm{t}_{\mathrm{av}} \mathrm{f}+1
$$

where $f$ is the actual sampling frequency. Beats whose length $\left(t_{i}\right)$ differed from $t_{a v}$ were recreated by using an adjustable sampling frequency $\left(\mathrm{f}_{\mathrm{i}}\right)$, such that the number $\left(\mathrm{N}_{\mathrm{i}}\right)$ of points in the resampled beat was exactly the same as $\mathrm{N}_{\mathrm{av}}$ as follows:

$$
f i=\frac{N_{a v}-1}{t_{i}}
$$

where an additive factor of 1 is again included to account for both end points of the beat. Values at each time step in the resampled beat were calculated by linearly interpolating between the 2 immediately surrounding points of the original beat.

With the length of all constituent beats correctly adjusted, an ensemble-averaging subroutine was used to calculate the algebraic mean of all pressure pulse signals point by point. The EC algorithm possesses a significant advantage over the traditional TE algorithm in that it preserves the overall periodicity and the details of the individual beats in the ensemble. Figure 1 shows a graphic comparison of the EC versus TE algorithms.

Mathematic modeling of $\boldsymbol{R} \boldsymbol{V}$ Relaxation. The exponential ${ }^{8,9,11-13}$ model (equation 2) represented well the time course of $\mathrm{RV}$ pressure decay. The RV pressure decay period was fixed as the interval from the beat beginning (defined above) up to the last point satisfying the following criteria: (1) it preceded the point of minimum RV diastolic pressure, and (2) its pressure level was at least $3 \mathrm{~mm} \mathrm{Hg}$ higher than the subsequent end-diastolic pressure $8,9,12,14$ for the case of RV pressure overload and $1 \mathrm{~mm} \mathrm{Hg}$ for control conditions, volume overload, and ischemia. Further analysis was restricted to this interval:

$$
\mathrm{P}_{\mathrm{R}}=\mathrm{P}_{0} \cdot \exp (-\mathrm{t} / \tau)+\mathrm{P}_{\mathrm{b}}
$$

The exponential model characterizes the decay of RV pressure $\left(\mathrm{P}_{\mathrm{R}}\right)$ with 3 parameters: $\mathrm{P}_{0}, \tau$, and $\mathrm{P}_{\mathrm{b}}$. Among them, $\tau$, the time constant of relaxation, is the most important because it quantifies the rate of relaxation. ${ }^{15} \mathrm{P}_{\mathrm{b}}$ is also important because it represents the asymptotic level to which the pressure would tend if the decaying process was allowed to proceed indefinitely. Previous studies have shown that the value of $\mathrm{P}_{\mathrm{b}}$ should not differ greatly from zero. If found to do so, it is a hallmark of the inapplicability of the monoexponential model as an accurate descriptor of relaxation dynamics. ${ }^{8,12,15}$ Finally, when the mean pleural pressure does not deviate significantly from zero, the parameter $\mathrm{P}_{0}$ is indicative of the beginning level of the exponential RV pressure decay. With the use of the nonlinear regression routine from the statistical software SPLUS, ${ }^{16}$ the experimental data were fitted to the exponential model, and least-squares parameter estimators were obtained.

\section{Statistics}

The exponential model parameters were used to assess the effect of each mode of RV failure on RV relaxation dynamics. For each parameter, a set of data was recorded under each condition (ie, control, pressure overload, volume overload, and ischemia). All data sets are presented as means $\pm \mathrm{SD}$. An analysis of variance for each variable was performed, with the 4 data groups being control $(n=15)$, pressure overload $(n=6)$, volume overload $(n=7)$, and ischemia $(n=7)$. Because the mean of each data set did not differ significantly from the median, the Student unpaired $t$ test was performed to assess statistical significance between the control and each of the RV disease models.

The $\alpha$ level for the Student $t$ test was adjusted with the Bonferronian inequality, thus using a more conservative critical value for the $t$ statistics to accommodate the fact that 3 comparisons were made against the control set for each variable.

The paired Student $t$ test was applied to determine whether values of the asymptote $\mathrm{P}_{b}$ in the conditions investigated were statistically different from zero.

\section{Results}

\section{Representative RV Hemodynamics}

Figure 2 contains 4 panels, each showing a train of representative steady-state RV pressure pulses. The most distinc- 


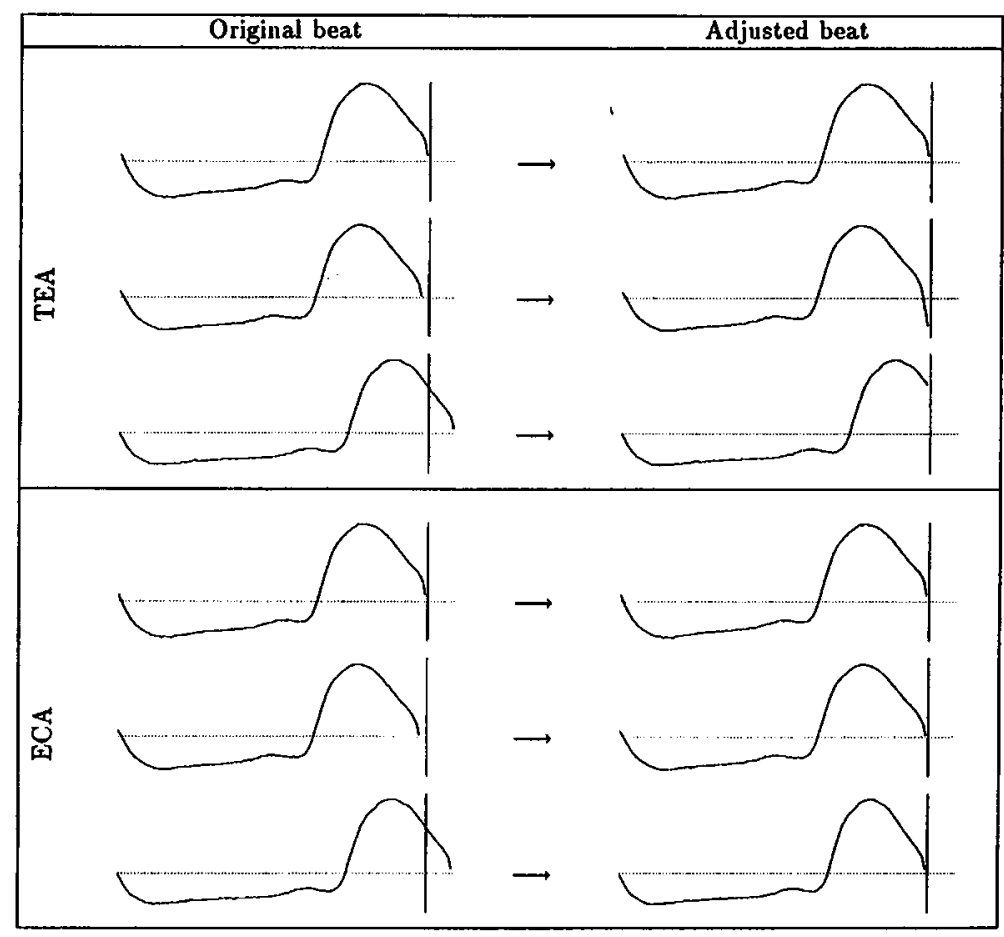

Figure 1. Comparison of TE and EC algorithms. The top panels show application of the TE algorithm to 3 waveforms: the second and third were extrapolated and truncated, respectively. The bottom panels show application of the EC algorithm to the same waveforms: all 3 beats preserve their original shapes.

tive difference between the tracings from pressure overload and control is in pressure level. Although under control conditions the measured $\mathrm{RV}$ pressure decreased to around 0 $\mathrm{mm} \mathrm{Hg}$, the lowest pressure under pressure overload remained greater than $10 \mathrm{~mm} \mathrm{Hg}$. In addition, peak systolic pressure was greater than $90 \mathrm{~mm} \mathrm{Hg}$ compared with less than $35 \mathrm{~mm} \mathrm{Hg}$ under control conditions.

Although the peak systolic pressure for volume overload was not significantly higher than that under control conditions, the minimum pressure was increased to about $10 \mathrm{~mm}$ $\mathrm{Hg}$. There was also a more pronounced pressure increase during filling: the difference between the nadir of the diastolic pressure and the peak of the atrial wave was about 5 $\mathrm{mm} \mathrm{Hg}$, approximately twice as much as that under control conditions.

The peak RV systolic pressure reached in ischemia was significantly depressed compared with that under control conditions. The magnitude of the pressure increase during filling, however, was similar to that under control conditions.

Figure 3 shows the mathematic modeling of RV relaxation dynamics. Data labeled as measured pressure in each of the 4 panels show the relaxation portion of the ensembleaveraged waveform. Data labeled as relaxation pressure show the tracing of the exponential model that best fits the measured pressure within the defined RV pressure decay period.

\section{Control Conditions}

The exponential model was applied to the RV relaxation process. Table 2 shows each parameter of the exponential model under control conditions. The paired Student $t$ test showed that $\mathrm{P}_{\mathrm{b}}$ values $(0.28 \pm 1.8 \mathrm{~mm} \mathrm{Hg}[$ mean $\pm \mathrm{SD}])$ did not differ from zero $(P>.54)$.

\section{Pressure Overload}

Dramatic increases in peak systolic pressure were observed (Figures 2 and 3). Table 2 shows the values of the exponential parameters for subacute (3-5 weeks) pressure overload. The increase in systolic pressure resulted in an increased $\mathrm{P}_{0}$. In addition, $\tau$ increased to $57 \pm 2.8 \mathrm{~ms}$ from $27.8 \pm 3.9 \mathrm{~ms}$ under control conditions $(P<.0001)$. This was accompanied by a general decrease in the value of $\mathrm{P}_{\mathrm{b}}$. However, $\mathrm{P}_{\mathrm{b}}$ remained well above $-10 \mathrm{~mm} \mathrm{Hg}$. Therefore, the exponential model remained applicable to pressure overload. ${ }^{8,12,15}$ However, $\mathrm{P}_{\mathrm{b}}$ was statistically less than $0 \mathrm{~mm} \mathrm{Hg}$ $(P<.0001)$. The SD values show that data for all 3 parameters fall into relatively narrow ranges. 

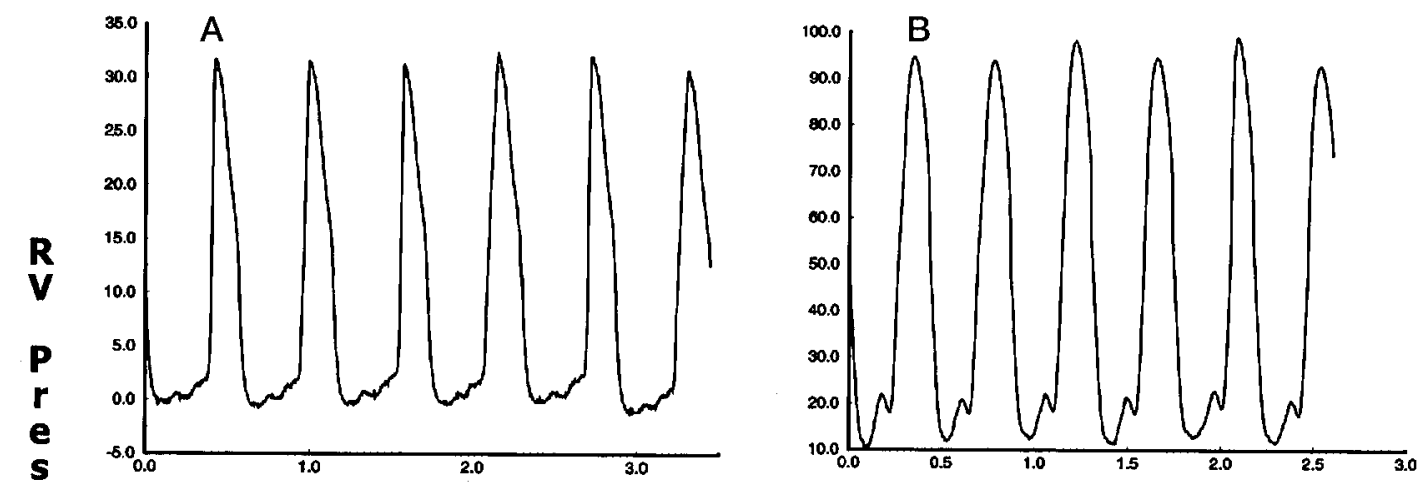

\section{Time ( $s$ )}
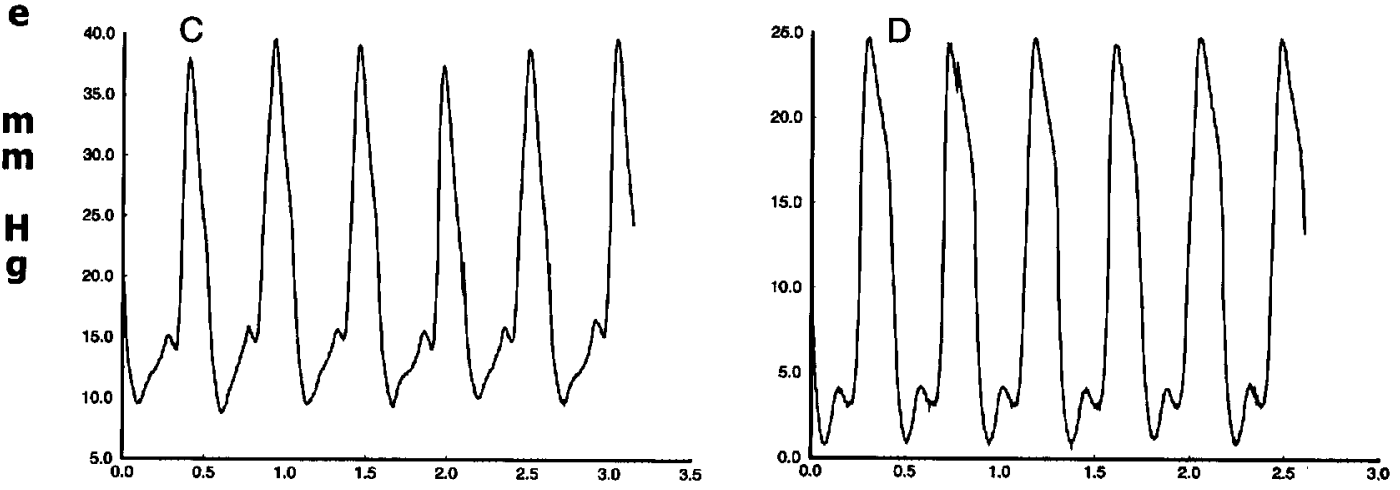

Figure 2. Steady-state RV pressure pulses under control conditions (A), pressure overload (B), volume overload (C), and ischemia (D). Peak systolic pressure is greatly increased under pressure overload, slightly increased under volume overload, and slightly decreased under ischemia.

\section{Volume Overload}

Table 2 summarizes the exponential parameters for subacute (2-4 weeks) volume overload. $\mathrm{P}_{0}$ does not show significant change from that under control conditions, which is consistent with no change in $\mathrm{RV}$ pressure at the onset of the relaxation period. The values of $\tau$ are likewise similar to those under control conditions. The level of $\mathrm{P}_{\mathrm{b}}(5.3 \pm 2.9$ $\mathrm{mm} \mathrm{Hg}$ [mean $\pm \mathrm{SD}]$ ) is statistically greater than $0 \mathrm{~mm} \mathrm{Hg}$ $(P<.001)$ and shows a significant increase from that under control conditions.

\section{Ischemia}

Table 2 summarizes the values of each parameter for subacute (1-2 weeks) ischemia. Unlike the 2 interventions already discussed, a significant reduction in peak RV systolic pressure was observed. The mean $\mathrm{P}_{0}$ was reduced to less than $10 \mathrm{~mm} \mathrm{Hg}$, which is consistent with the general depression of RV pressure. The $\tau$ value, however, was increased significantly $(P<.0004)$. For ischemia, the mean $\tau(41.4 \pm 13.0 \mathrm{~ms})$ falls in between the means under control conditions and pressure overload. $\mathrm{P}_{\mathrm{b}}(-1.95 \pm 1.11 \mathrm{~mm} \mathrm{Hg}$ [mean $\pm \mathrm{SD}])$ is decreased from that at the control condi- tion and is now less than $0 \mathrm{~mm} \mathrm{Hg}(P<.001) . \mathrm{P}_{\mathrm{b}}$ values during ischemia were uniformly negative. Similar to $\tau$, the level of $\mathrm{P}_{\mathrm{b}}$ under ischemia was between those under control conditions and under pressure overload.

\section{Statistical Analysis of the Effect of RV Disease Models on Relaxation}

The bar charts in Figure 4 show descriptive statistics for the exponential model coefficients under control conditions, pressure overload, volume overload, and ischemia. Bar height and error brackets indicate means and SE, respectively. Table 2 displays analysis of variance $\mathrm{F}$ statistics and associated $P$ values. Also included are results of Student $t$ tests comparing the mean of each exponential coefficient in each disease state with that under control conditions. Results of the Student $t$ tests that are significant under the Bonferronian inequality are presented in boldface.

Pressure overload differs significantly from control conditions in all 3 parameters: there are increases in both $\mathrm{P}_{0}$ and $\tau$ and a decrease in $\mathrm{P}_{\mathrm{b}}$. Volume overload, on the other hand, displays only a significant increase in $\mathrm{P}_{\mathrm{b}}$. This is consistent with the observations made above on the relatively high 

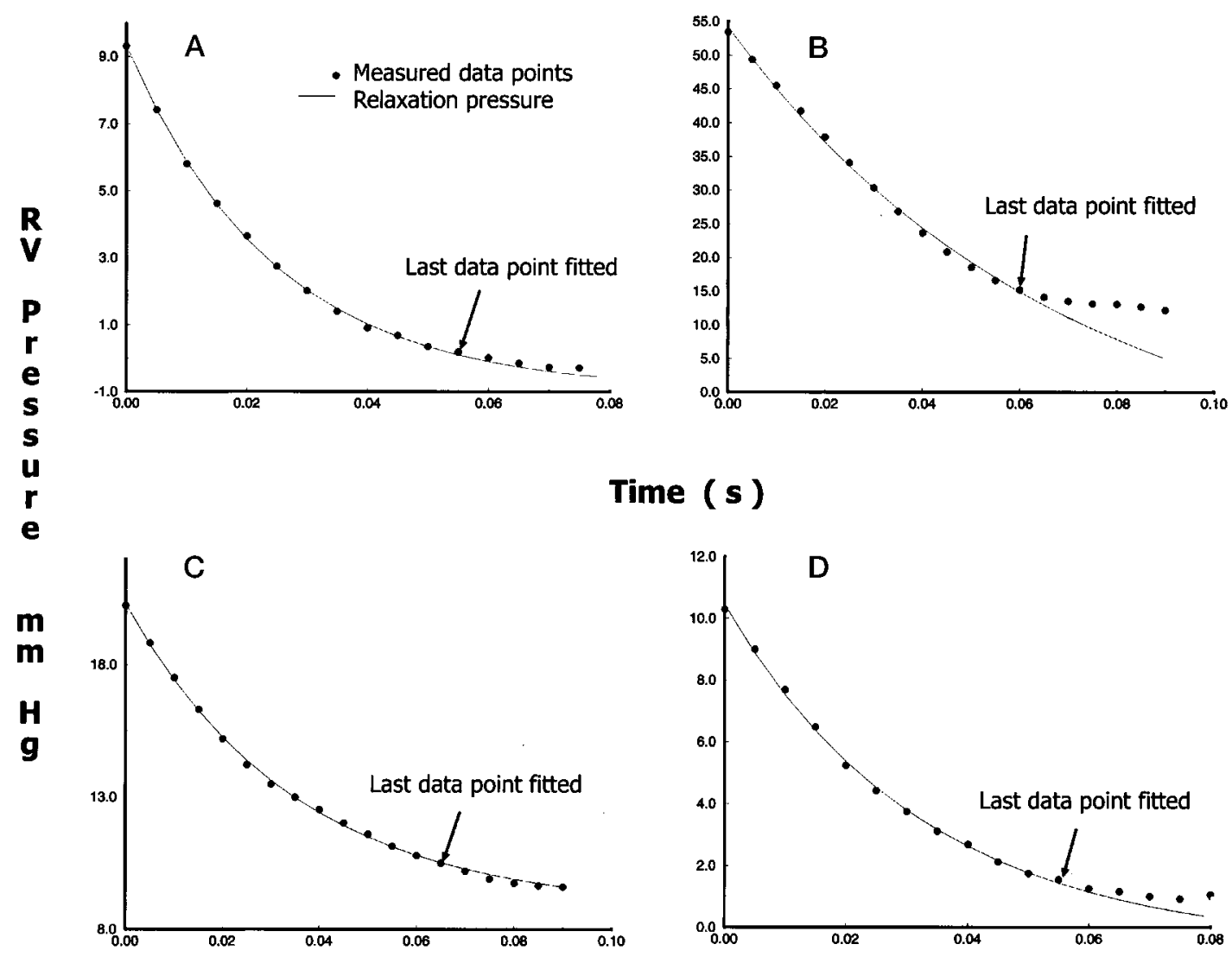

\section{Time ( $s$ )}

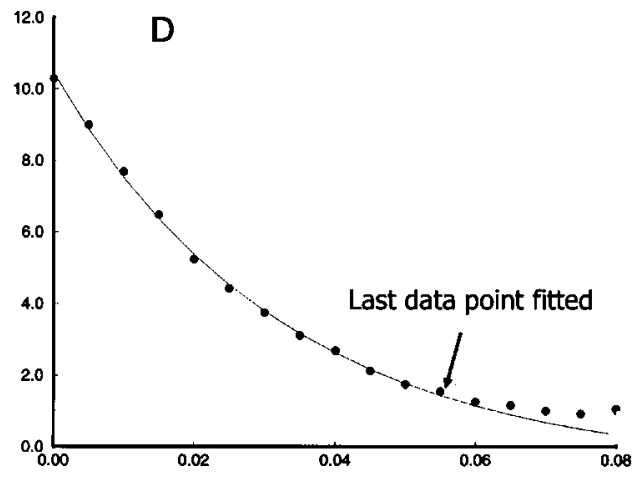

Figure 3. Tracings of RV isovolumic pressure decay under control conditions (A), pressure overload (B), volume overload (C), and ischemia (D). Dots denote every other point in the digital ensemble average of the measured waveforms. The line shows the exponential fit. Arrows indicate the last points used in regression.

TABLE 2. Isovolumic relaxation dynamics

\begin{tabular}{|c|c|c|c|c|}
\hline & & $P_{0}(m m ~ H g)$ & $P_{b}(m m ~ H g)$ & $\tau(\mathrm{ms})$ \\
\hline \multirow[t]{4}{*}{ Condition (mean \pm SD) } & Control & $13.1 \pm 5.1$ & $0.28 \pm 1.8$ & $27.8 \pm 3.9$ \\
\hline & $\mathrm{PO}$ & $56.2 \pm 19.1$ & $-7.9 \pm 1.5$ & $57.1 \pm 2.8$ \\
\hline & VO & $11.3 \pm 5.1$ & $5.3 \pm 2.9$ & $26.8 \pm 6.1$ \\
\hline & Ischemia & $9.0 \pm 3.5$ & $-1.95 \pm 1.1$ & $41.4 \pm 13.0$ \\
\hline \multirow[t]{2}{*}{ Analysis of variance } & F statistic & 43.33 & 51.62 & 31.81 \\
\hline & $P$ value & .0001 & .0001 & .0001 \\
\hline \multirow[t]{3}{*}{$\mathrm{P}_{\text {BONF }}{ }^{*}=.0167$} & PO vs control & .0001 & .0001 & .0001 \\
\hline & VO vs control & .2295 & .0001 & .3140 \\
\hline & Ischemia vs control & .0355 & .0038 & .0004 \\
\hline
\end{tabular}

$S D$, Standard deviation; $P O$, pressure overload; VO, volume overload. Values significantly different from control are presented in boldface.

*ANOVA with Bonferroni's inequality. For the 3 comparisons, a conservative critical value for the modified $t$ statistics is given by using a significance level, $\mathrm{P}_{\mathrm{BONF},}$ of $(\mathrm{P} / 3)=(.05 / 3)=.0167$.

pressure at the nadir of the RV diastolic pressure and the lack of significant changes from control conditions in $\mathrm{P}_{0}$ and $\tau$. $\mathrm{RV}$ ischemia is associated with a significant increase in $\tau$ and a decrease in $\mathrm{P}_{\mathrm{b}}$. The directions of change for $\tau$ and $\mathrm{P}_{\mathrm{b}}$ are identical to those under pressure overload. However, the magnitudes of the changes are greater for pressure overload. There is also evidence $(P=.035)$ of a significant decrease in $\mathrm{P}_{0}$ under $\mathrm{RV}$ ischemia, which is consistent with the reduced peak systolic pressure (Figures 2 and 3 ).

\section{Discussion}

Using a combination of newly developed surgical chronic animal models and mathematic analyses, the present study compared, for the first time, relaxation dynamics in the 


\section{Comparison of P0}

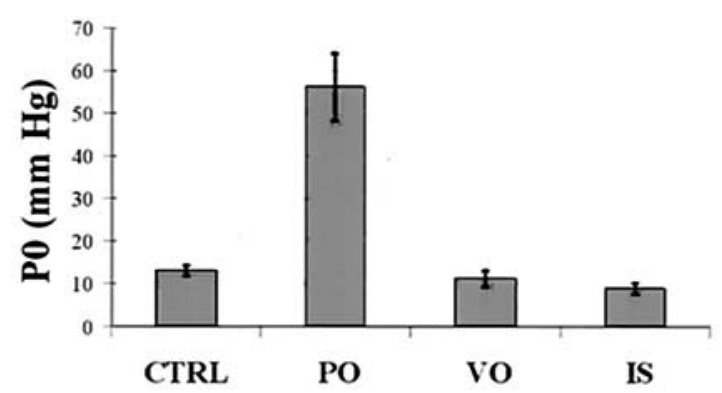

\section{Comparison of tau}

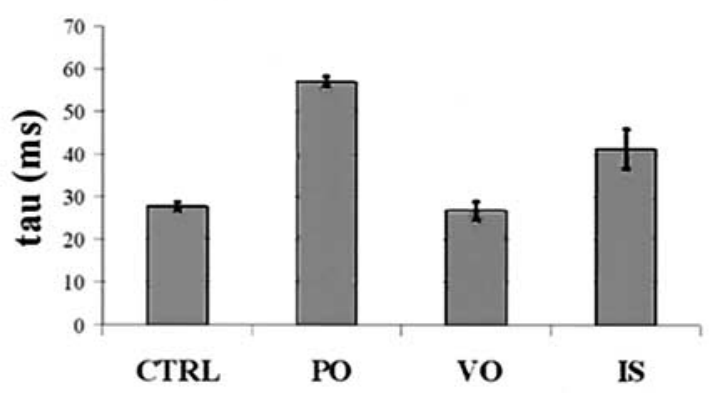

Comparison of $\mathbf{P b}$

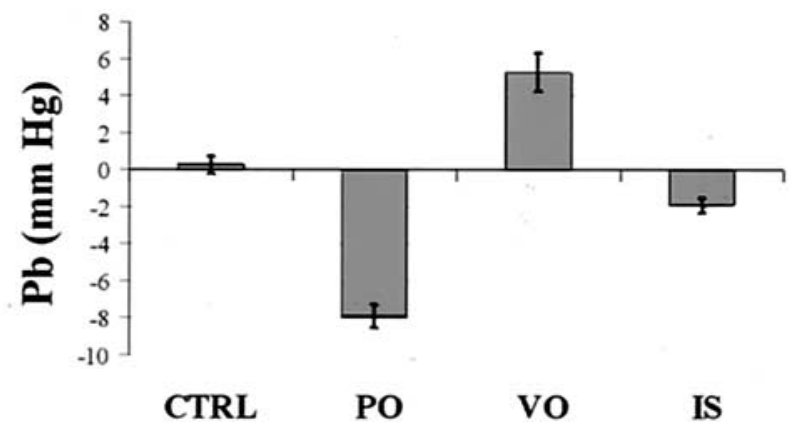

Figure 4. Descriptive statistics of the exponential model coefficients. CTRL, Control condition; PO, pressure overload; VO, volume overload; IS, ischemia.

normal and chronically diseased right ventricles of awake dogs to characterize important abnormalities resulting from both pressure and volume overload and from RV free wall ischemia.

\section{RV Applicability of the Exponential Model With \\ Asymptote}

In the analysis of RV relaxation mechanics, we demonstrated the general applicability of the monoexponential model with asymptote 8,15 in describing the characteristics of $\mathrm{RV}$ pressure decay. In the case of the left ventricle, it was previously recommended that the lower cutoff pressure used in the regression should exceed the subsequently attained end-diastolic pressure by $3 \mathrm{~mm} \mathrm{Hg.,9,12,14} \mathrm{This} \mathrm{recommen-}$ dation was followed in the analysis of RV pressure overload, when the range of operating pressure is similar to that in the left ventricle. For the analysis of control, volume overload, and ischemia, we adjusted the cutoff level to at least $1.0 \mathrm{~mm} \mathrm{Hg}$ greater than the subsequent end-diastolic pressure level. Because the RV operating pressure range is significantly lower, this adjustment from earlier LV applications was needed to have sufficient data points for the regression. In no case were there fewer than 23 points available for curve-fit parameter estimation; there were at least 7 points per estimated model coefficient.
We validated the suitability of the 3-parameter exponential model for RV use by applying it successfully to normal and subacute animal models simulating RV dysfunction states with distinct causes. Craig and colleagues ${ }^{15}$ introduced the nonzero pressure asymptote and showed that forcing a zero asymptote can yield spurious changes in $\tau$. Care should be taken in determining circumstances when the monoexponential model is actually inadmissible. ${ }^{8,12,15}$ Most notably, caution must be exercised when $\mathrm{P}_{\mathrm{b}}$ is found to deviate greatly from zero because this is a hallmark of the nonapplicability of the monoexponential model as an accurate descriptor of relaxation dynamics.

\section{RV Relaxation Abnormalities in Pressure Overload, Volume Overload, and Ischemia}

For control, $\mathrm{P}_{\mathrm{b}}$ in the right ventricle was not found to be significantly different from zero $(P>.54)$. This result is in agreement with studies on the left ventricle. ${ }^{9,11-13}$ Considering the $3 \mathrm{RV}$ disease states, however, we found that $\mathrm{P}_{\mathrm{b}}$ increased significantly in RV volume overload $(P<.0001)$ and decreased in pressure overload and ischemia $(P<$ .001). These findings indicate that for the right ventricle, as for the left, the exponential model is well suited to describing RV pressure decay under normal (control) conditions, regardless of whether it allows for a nonzero asymptote or 
not. However, to describe the decay under subacute pressure overload, volume overload, and ischemia, the exponential with nonzero asymptote is appropriate.

Previous studies on LV ischemia ${ }^{8,13}$ have shown that impairment of relaxation is often associated with a lengthening of $\tau$. The same pattern was observed in our RV study for both pressure overload and ischemia. The prolongation of $\tau$ in ischemia might reflect the decreased rate of $\mathrm{Ca}^{++}$ reuptake by the sarcoplasmic reticulum, which is an active process. ${ }^{8}, 12,13,15,17$ Because during RV pressure overload the hypertrophied right ventricle has to overcome an increased systolic load during ejection, RV myocardial perfusion might fail to increase sufficiently to satisfy the increased RV myocardial mass and metabolic demand. This might result in regional subendocardial ischemia of the RV free wall. ${ }^{18}$ Our results on the right ventricle agree with those of previous studies on the left ventricle. ${ }^{8}$ The decrease in $\mathrm{P}_{\mathrm{b}}$ in ischemia might result from increased sympathetic tone. ${ }^{8,11}$

In contrast to the results obtained for RV pressure overload and ischemia, no significant lengthening of $\tau$ was found for volume overload. This result suggests that the relaxation mechanism is unimpaired in subacute volume overload. It correlates with previous empiric observations ${ }^{1}$ that the right ventricle, as a thin-walled, low-pressure system, tolerates an increase in the preload significantly better than it tolerates increases in the afterload. The only significant change detected by the model in RV volume overload is the increase of the asymptote, $\mathrm{P}_{\mathrm{b}}$. This reflects increased diastolic constraint because of the elevated right-heart volumes. ${ }^{7,8,14}$

\section{Clinical Significance}

This study is clinically important because it is the first to demonstrate several abnormalities of RV relaxation, which might have clinical significance and implications for patient management. ${ }^{19}$ A previous acute surgical experimental study ${ }^{20}$ on open-chest anesthetized dogs had reported absence of isovolumic relaxation, which appears to be referable to the applying highly unphysiologic state and methods. Ihara and coworkers ${ }^{11}$ have shown, in elegant experiments, that in the anesthetized state immediately after the chest was closed after instrumentation procedures, ejection time was greatly prolonged compared with that during control awake conditions, whereas left ventricular end-systolic stress and mean systolic arterial pressure were reduced substantially. Together, these factors act to factitiously encroach on the isovolumic relaxation period. This effect should be similar on the right side and enhanced in the open-chest state because of a lack of the RV afterload component represented in the intact awake state by the action of pleuropericardial adhesive forces and lung elasticity, creating negative intrathoracic pressure. In a human multisensor catheterization study, Condos and coworkers ${ }^{7}$ showed that under awake conditions, conventional RV systolic ejection period was $371 \pm 17 \mathrm{~ms}$ (mean $\pm \mathrm{SD})$, whereas RV flow time (catheter-mounted electromagnetic flow sensor) was only $298 \pm 19 \mathrm{~ms}$. Considering that their onset is simultaneous, there is substantial underestimation (by $73 \mathrm{~ms}$ on average) of the true extent of the isovolumic relaxation period if its onset is incorrectly assumed to be the end of the conventional systolic ejection period (incisural nadir).

The impairment of RV relaxation (increased $\tau$ ) demonstrated in pressure overload and ischemia suggests that the pressure-overloaded or ischemic right ventricle might be less tolerant of tachycardia than the normal or volumeoverloaded right ventricle. In pressure overload or ischemia, tachycardia would cause disproportionately large increases in RV diastolic pressure, which in turn would accentuate wall stress and central venous pressures. By reducing the heart rate, myocardial oxygen consumption would decrease, resulting in a better relationship between supply and demand; this is true regardless of whether there is epicardial coronary disease (ischemia) or whether there is just hypertrophy and subendocardial ischemia (pressure overload). In pressure overload, treatment should be aimed at normalizing load and preventing or regressing (or both) RV hypertrophy. In ischemia it should be aimed at decreasing myocardial oxygen consumption (demand) and increasing myocardial blood flow (supply). Both catheter-based and surgery-based methods of revascularization have been shown to improve left-sided diastolic dysfunction. ${ }^{19}$

On the other hand, in volume overload, RV pressures during relaxation are disproportionately augmented by an increased pressure asymptote $\left(\mathrm{P}_{\mathrm{b}}\right)$. This suggests that the volume-overloaded right ventricle experiences a more constant elevation of diastolic pressure that would be independent of heart rate and that might result from or be accentuated by pericardial constraint. Increase of $\mathrm{P}_{\mathrm{b}}$ might therefore contribute to less tolerance of pericardial constraint or scarring in RV volume overload than in pressure overload or ischemia. Increased RV diastolic pressure during exercise might also limit subendocardial blood flow during increased myocardial oxygen demand, worsening diastolic dysfunction. As indicated in the setting of LV diastolic failure, ${ }^{19}$ treatment should be aimed at normalizing RV volume by increasing venous capacitance with nitrates, nitroprusside, or morphine; fluid and sodium restriction; or surgical intervention.

\section{Conclusions}

We conclude that significant abnormalities in the dynamics of $\mathrm{RV}$ relaxation result from both pressure and volume overload and from RV free wall ischemia. These changes, demonstrated in animal models of RV dysfunction of distinct causes, and their interactions with associated alterations in RV passive diastolic properties ${ }^{21}$ should influence $^{8,21}$ filling dynamics in RV dysfunction and are in need of further investigation. 


\section{References}

1. Barnard D, Albert JS. Right ventricular function in health and disease. Curr Probl Cardiol. 1987;12:417-49.

2. Calvin JE. Right ventricular diastolic function after experimental right ventricular infarction: effects independent of the pericardium. Clin Invest Med. 1991;14:346-54.

3. Goldstein JA, Barzilai B, Rosamond T, Eisenberg P, Jaffe A. Determinants of hemodynamic compromise with severe right ventricular infarction. Circulation. 1990;82:359-68.

4. Lee FA. Hemodynamics of the right ventricle in normal and disease states. Cardiol Clin. 1992;10:59-65.

5. Fridl P, Horak J, Fabian J, Kokandrle V. Right ventricle in patients after orthotopic heart transplantation. Cor Vasa. 1990;32:206-10.

6. Hines R. Right ventricular function and failure: a review. Yale J Biol Med. 1991;64:295-307.

7. Condos WR, Latham RD, Hoadley SD, Pasipoularides A. Hemodynamic effects of the Mueller maneuver by simultaneous right and left heart micromanometry. Circulation. 1987;76:1020-8.

8. Mirsky I, Pasipoularides A. Clinical assessment of diastolic function. Prog Cardiovasc Dis. 1990;32:291-318.

9. Pasipoularides A, Mirsky I, Hess OM, Grimm J, Krayenbuehl HP. Myocardial relaxation and passive diastolic properties in man. Circulation. 1986;74:991-1001.

10. Pasipoularides A. Clinical assessment of ventricular ejection dynamics with and without outflow obstruction. $J$ Am Coll Cardiol. 1990;15: 859-82.

11. Ihara T, Shannon RP, Komamura K, Pasipoularides AD, Patrick T, Shen S, Vatner SF. Effects of anaesthesia and recent surgery on diastolic function. Cardiovasc Res. 1994;28:325-36.
12. Pasipoularides A, Mirsky I. Models and concepts of diastolic mechanics: pitfalls in their misapplication. Math Comp Model. 1988;11:232-4.

13. Paulus WJ, Grossman W, Serizawa T, Bourdillon PD, Pasipoularides AD, Mirsky I. Different effects of two types of ischemia on myocardial systolic and diastolic function. Am J Physiol. 1985;248:H719-28.

14. Gilbert J, Glantz, S. Determinants of left ventricular filling and of the diastolic pressure-volume relationship. Circ Res. 1989;64:827-52.

15. Craig WE, Murgo JP, Pasipoularides A. Evaluation of time course of left ventricular isovolumic relaxation in humans. In: Grossman W, Lorell BH, eds. Diastolic relaxation of the Heart. Boston: Martinus, Nijhoff; 1988. p. 125-32.

16. Chambers JM, Bates DM. Nonlinear models. In: Chambers DM, Hastie TJ, eds. Statistical models in S. New York: Chapman \& Hall; 1990. p. 421-55.

17. Palacios I, Newell JB, Powell WJ. Effect of acute global ischemia on diastolic relaxation in canine hearts. Am J Physiol. 1978;235:H720-7.

18. Murray PA, Vatner SF. Reduction of maximal coronary vasodilator capacity in conscious dogs with severe right ventricular hypertrophy. Circ Res. 1981;48:25-33.

19. Zile MR, Simsic JM. Diastolic heart failure: diagnosis and treatment. Clin Cornerstone. 2000;3:13-24.

20. Myhre ES, Slinker BK, LeWinter MM. Absence of right ventricular isovolumic relaxation in open-chest anesthetized dogs. Am J Physiol. 1992;263:H1587-90.

21. Pasipoularides AD, Shu M, Shah A, Silvestry S, Glower DD. Right ventricular diastolic function in canine models of pressure overload, volume overload and ischemia. Am J Physiol: Heart Circ Physiol. In press. Published online ahead of print, July 18, 2002 10.1152/ajpheart.00462.2002.

\section{Availability of Journal back issues}

As a service to our subscribers, copies of back issues of The Journal of Thoracic and Cardiovascular Surgery for the preceding 5 years are maintained and are available for purchase from Mosby until inventory is depleted. Please write to Mosby, Subscription Customer Service, 6277 Sea Harbor Dr, Orlando, FL 32877, or call $800-654-2452$ or $407-345-4000$ for information on availability of particular issues and prices. 